\title{
Chronic stress promotes EMT-mediated metastasis through activation of STAT3 signaling pathway by miR-337-3p in breast cancer
}

\author{
Peixin Du' ${ }^{1}$, Hao Zeng ${ }^{1}$, Yinan Xiao ${ }^{1}$, Yunuo Zhao ${ }^{1}$, Bo Zheng ${ }^{1}$, Yaotiao Deng ${ }^{1}$, Jie Liu' ${ }^{1}$ Boyan Huang ${ }^{1}$, Xinyao Zhang ${ }^{1}$,
} Keyi Yang ${ }^{1}, Y u$ Jiang ${ }^{1}$ and Xuelei $\mathrm{Ma}^{1}$

\begin{abstract}
Chronic stress could induce cancer metastasis by constant activation of the sympathetic nervous system, while cellular mechanism remains obscure. The aim of this research is to explore the metastasis associated negative effect of chronic stress. The analysis of transcriptome sequencing implied that activation of STAT3 signaling pathway by downregulated miR-337-3p might be a potential mechanism to induce epithelial to mesenchymal transition (EMT) of cancer cell and promote metastasis under chronic stress. We also verified this biological process in further experiments.

Downregulation of miR-337-3p could downregulate E-cadherin expression and upregulate vimentin expression in vitro and in vivo. STAT3, related signal pathways of which are involved in metastasis regulation, was directly targeted by miR-337-3p. In conclusion, the above results denoted that activation of miR-337-3p/STAT3 axis might be a potential pathway for the increasing metastasis of breast cancer under chronic stress.
\end{abstract}

\section{Introduction}

Psychological and emotional problems arise as a result of long-term challenges to physical and mental health and low quality of life in cancer patients ${ }^{1}$. With the development of research in psychological disorders of cancer patients, accumulating evidence showed that psychosocial stress could influence the development, progression, and metastasis of cancer ${ }^{2,3}$. Breast cancer remains the most common cancer in women worldwide and metastasis is widely accepted as the major cause for cancer death ${ }^{4,5}$. In breast tumor bearing mice model, psychosocial stress could induce the formation of pre-metastatic niches in distant organs ${ }^{6-9}$, while blocking stress-associated hormone factors could reduce the metastasis ${ }^{10,11}$. Previous clinical research also indicated that stress-related factors are involved in promoting breast cancer progression ${ }^{12}$.

\footnotetext{
Correspondence: Yu Jiang (jiang_yu@scu.edu.cn) or

Xuelei Ma (drmaxuelei@gmail.com)

'Department of Medical Oncology, Cancer Center, State Key Laboratory of

Biotherapy, West China Hospital, Sichuan University, Sichuan, China

Edited by R. Aqeilan
}

Therefore, a better understanding of underlying mechanism serves to prevent the negative effect of chronic stress on metastasis.

One of the key molecular mechanisms contributing to the metastatic progression is epithelial to mesenchymal transition (EMT), which drives invasion and migration of various cancer including breast cancer ${ }^{13}$. EMT, a conserved cellular program bestowing mesenchymal-like traits on epithelial cells, is often accompanied by changes in expression level of E-cadherin and vimentin ${ }^{14}$. In a clinical research, human breast carcinoma tissues with lower E-cadherin expression could predict poor metastatic-free survival outcome ${ }^{15}$.

miRNAs are short, 18-25 nucleotide-long, noncoding RNA molecules, and they exert impact on cell proliferation, differentiation, and apoptosis ${ }^{16}$. miRNAs could suppress gene expression at both transcriptional and posttranscriptional level by binding to complementary sequences of its target mRNA ${ }^{17}$. miRNAs serve as a crucial components in epigenetic modification for many important biological pathways including $\mathrm{EMT}^{18}$. Previous

\section{(c) The Author(s) 2020}

(c) Open Access This article is licensed under a Creative Commons Attribution 4.0 International License, which permits use, sharing, adaptation, distribution and reproduction cc) in any medium or format, as long as you give appropriate credit to the original author(s) and the source, provide a link to the Creative Commons license, and indicate if changes were made. The images or other third party material in this article are included in the article's Creative Commons license, unless indicated otherwise in a credit line to the material. If material is not included in the article's Creative Commons license and your intended use is not permitted by statutory regulation or exceeds the permitted use, you will need to obtain permission directly from the copyright holder. To view a copy of this license, visit http://creativecommons.org/licenses/by/4.0/. 
research has suggested that miR-337-3p could regulate the EMT process during cancer progression and metastasis $^{19}$. Several signaling pathway including STAT3 pathway has been reported to participate in the development of EMT process in breast cancer ${ }^{20}$. Previous studies revealed the negative targeting relationship between miR337-3p and JAK2/STAT3 and further demonstrated that STAT3 is directly targeted by miR-337-3p $\mathrm{p}^{21,22}$. Additionally, accumulating evidence has validated that miRNAs expression is often dysregulated by stress in cancer, such as chronic stress ${ }^{23}$, heat stress $^{24}$, and surgical stress $^{25}$. The rapid development of sequencing technology enables the detection of genetic changes in mutated cells, which promote the understanding for cancer biology ${ }^{26,27}$. In comparison to the normal control, chronic stress may lead to 20 upregulated miRNAs and 35 downregulated miRNAs detected by using miRNA microarrays method ${ }^{28}$.

In this study, the next-generation sequencing analysis was used to investigate the biological function of dysregulated miRNAs induced by chronic stress in cancer metastasis. Then, we selected miR-337-3p according to sequencing profiles and biological informatics analysis for further study. Finally, we discovered that inhibited expression of miR-337-3p and its downstream target STAT3 might regulate EMT and promote metastasis in breast cancer models suffering chronic stress.

\section{Results}

Chronic stress promoted breast cancer metastasis in vivo

In order to evaluate the contribution of chronic stress to breast tumor growth and metastasis, $1 \times 10^{5} 4 \mathrm{~T} 1$ cells were subcutaneously inoculated into the right breast region of 6-weeks-old female $\mathrm{BALB} / \mathrm{c}$ mice to form xenograft model. We observed no significant difference in tumor growth curve and tumor volume between stress group and control group (Fig. 1a, b). However, pulmonary metastasis was more detectable in the mice of stress group (Fig. 1c). Notably, we found that chronic restraint stress increased the number of lung metastatic nodules, especially for those with width less than $2 \mathrm{~mm}$ (Fig. 1d). To sum up, we verified that chronic restraint stress contributed to lung metastasis of breast cancer without promoting its growth.

\section{Chronic stress and norepinephrine (NE) induced EMT in breast cancer in vivo and vitro}

To determine whether chronic stress contributed to the invasive phenotype of the cancer cells by inducing EMT, we resorted to immunohistochemical staining and western blot analysis to determine the altered expression of EMT-related markers in tumor tissue removed from the mice of two groups above. The results of immunohistochemical staining indicated downregulation of E-cadherin and upregulation of vimentin in stress group compared with control group (Fig. 2a, b), which confirmed the development of EMT in 4T1 breast cancer cells. Furthermore, the western blot analysis demonstrated similar results (Fig. 2c-f).

As one of the most important stress-related hormones, NE was widely used to mimic the effect of chronic stress in vitro experiment. In wound healing assay, after culturing with $10 \mu \mathrm{M} \mathrm{NE}$, 4T1 cells showed faster migratory speed in comparison with the cells in control group (Fig. $2 \mathrm{~g}, \mathrm{~h}$ ). Furthermore, we observed that cells incubated with NE became more slender and separated, which was consist with the characteristics of mesenchymal cells induced by TGF- $\beta$, while cells in control group showed more epithelial cells features under the light microscope (Fig. 2i). Moreover, immunofluorescence staining demonstrated that expression level of E-cadherin was downregulated and the expression level of vimentin was upregulated in cell cultured with NE (Fig. 2j, k) in vitro and similar observations were obtained in western blot analysis (Fig. 2l-o). Taken together, these results showed that chronic stress and NE induced EMT of 4T1 breast cancer in vivo and in vitro.

\section{miR-337-3p was downregulated by chronic stress and mediated EMT in 4T1 cells}

To figure out the critical role of miRNA in promoting the lung metastasis of breast cancer, we performed miRNA-sequencing on tumor tissues of mice in stress group and control group followed by differentially expressed (DE) analysis. By comparing differentially expressed genes between tumors in two groups, 22 aberrantly upregulated and 41 downregulated candidate genes $(P<0.05)$ were identified in stress group (Fig. 3a, b). Among these, 7 miRNAs (miR-397-3p, miR-540-3p, miR337-3p, miR-380-3p, miR-6240, miR-5121, and miR3068-3p) with $\mid \lg$ FC $\mid>1$ were selected (Fig. 3c). The RTqPCR analysis on miRNAs of 4T1 cells incubated with NE defined miR-337-3p as a possible factor in mediating the chronic stress associated metastasis (Fig. 3d).

To determine the effect of miR-337-3p on NE regulated EMT progression, miR-337-3p mimics (miR-337-3p-mi) was transfected into cultured $4 \mathrm{~T} 1$ cells to overexpress endogenous miR-337-3p, and transfection efficiency was detected by using RT-qPCR analysis (Fig. 3e). Furthermore, miR-337-3p-mi or negative control mimic (NC-mi) was transfected into $4 \mathrm{~T} 1$ cells cultured with NE. Immunofluorescence and western blot analysis displayed that both the decreased expression level of E-cadherin and elevated expression level of vimentin induced by NE were suppressed in cells transfected with miR-337-3p-mi (Fig. $3 \mathrm{f}-\mathrm{k}$ ). Taken together, aforementioned information validated that inhibited expression of miR-337-3p might be responsible for acquisition of EMT and cell migration in 4T1 cells cultured with NE. 
A

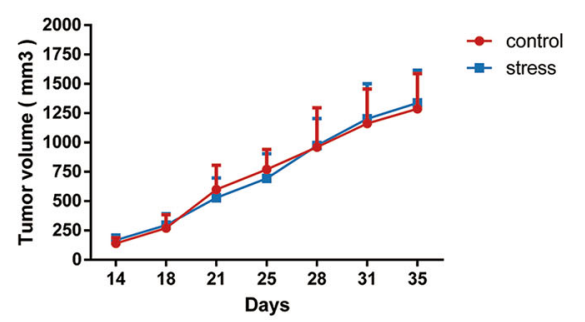

C

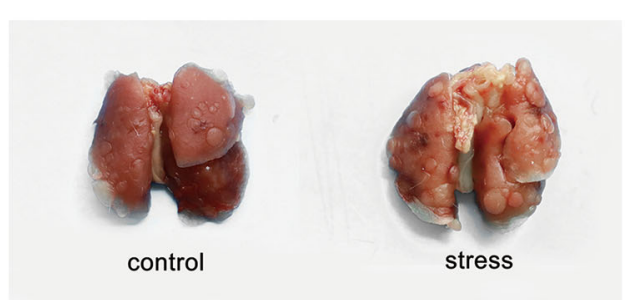

B

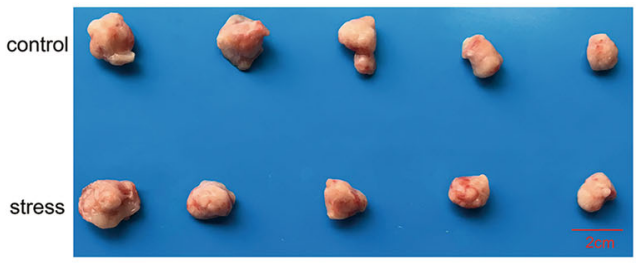

D

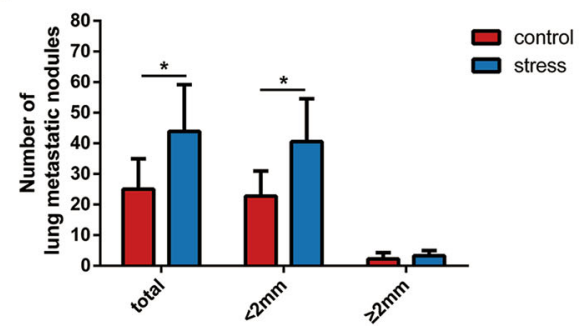

Fig. 1 Effect of chronic stress on 4T1 breast cancer in vivo. a Quantification of 4T1 primary tumor size in mice of control group and stress group over time $(n=5)$. $\mathbf{b}$ Images of the tumor xenografts from the control group and stress group are displayed 35 days after cell inoculation. $\mathbf{c}$ Representative lung images from the control group and stress group. $\mathbf{d}$ The histogram was used to compare the average numbers of metastatic nodules in the lungs from the control group and the stress group $(n=5)$. Data are shown as the mean $\pm S D$; $P<0.05$.

\section{miR-337-3p mediated EMT in 4T1 cells by directly targeting STAT3}

To identify the target mRNA of miR-337-3p involved in EMT progress of 4T1 cells, we also performed mRNAsequencing on the same tumor tissues from stress group and control group followed by DE analysis. By comparing 374 upregulated genes $(P<0.05)$ in stress group with the mRNAs related to miR-337-3p predicted in three data bases (DIANA tools, Starbase and Target scan), nine genes were screened out (Fig. 4a, b). Moreover, correlation heat map indicated that the nine genes were highly relevant to miR-337-3p (Fig. 4c) and STAT3 was selected for further study as previous evidence proved that it was targeted by miR-337-3 $\mathrm{p}^{29,30}$, and played a crucial role in $\mathrm{EMT}^{31}$.

To investigate whether STAT3 was regulated by NE/ miR-337-3p, we used western blot to explore the effect of NE and miR-337-3p on the expression of STAT3 and Phospho-STAT3-Tyr705 (p-STAT3) in 4T1 cells. The results showed that miR-337-3p overexpression by mimics inhibited STAT3 expression as well as p-STAT3 compared with that of group transfected with NC-mi (Fig. $4 d-g)$. We also found that, as expected, NE upregulated STAT3 and p-STAT3 levels, but this effect was suppressed in the cells overexpressing miR-337-3p (Fig. $4 d-g)$. These results demonstrated that STAT3 was a downstream molecule of NE/miR-337-3p signaling pathway.

To determine if STAT3 was directly regulated by miR337-3p, we used TargetScan to predict the conserved
miR-337-3p targeting sites located at $3^{\prime}$-UTR of STAT3 mRNA (Fig. 4h). The luciferase reporter gene assay indicated that after co-transfecting with miR-337-3p mimic and STAT3-WT, the luciferase activity of $4 \mathrm{~T} 1$ cells was remarkably decreased in contrast to the cells cotransfected with the negative control mimic and STAT3-WT. However, luciferase activity of STAT3-Mut co-transfection system showed no significant difference (Fig. 4i). The above results revealed that STAT3 gene was directly targeted by miR-337-3p.

To evaluate STAT3 mediated the effect of NE on EMT in $4 \mathrm{~T} 1$ cells, siRNAs were used to knocked down the gene. We applied three different STAT3 siRNAs and selected STAT3-siRNA1 and STAT3-siRNA2 for following experiments based on their better ability to inhibit the STAT3 mRNA (Fig. 4j). In contrast to control group, the increased expression of E-cadherin and decreased expression of vimentin in STAT3-siRNA1 and STAT3-siRNA2 group implied the reverse process of EMT (Fig. 4k, 1). Besides, STAT3-siRNA1 and STAT3-siRNA2 had an effect on reversing the EMT progression induced by NE (Fig. $4 \mathrm{~m}, \mathrm{n}$ ). The results above confirmed that STAT3 was a significant molecule negatively regulated by $\mathrm{miR}-337-3 \mathrm{p}$ and contributed to the progression of EMT.

\section{miR-337-3p inhibited lung metastasis and EMT of 4T1 tumor cells in vivo}

To explore the effect of miR-337-3p downregulation on tumor growth and metastasis in vivo, antagomir was 


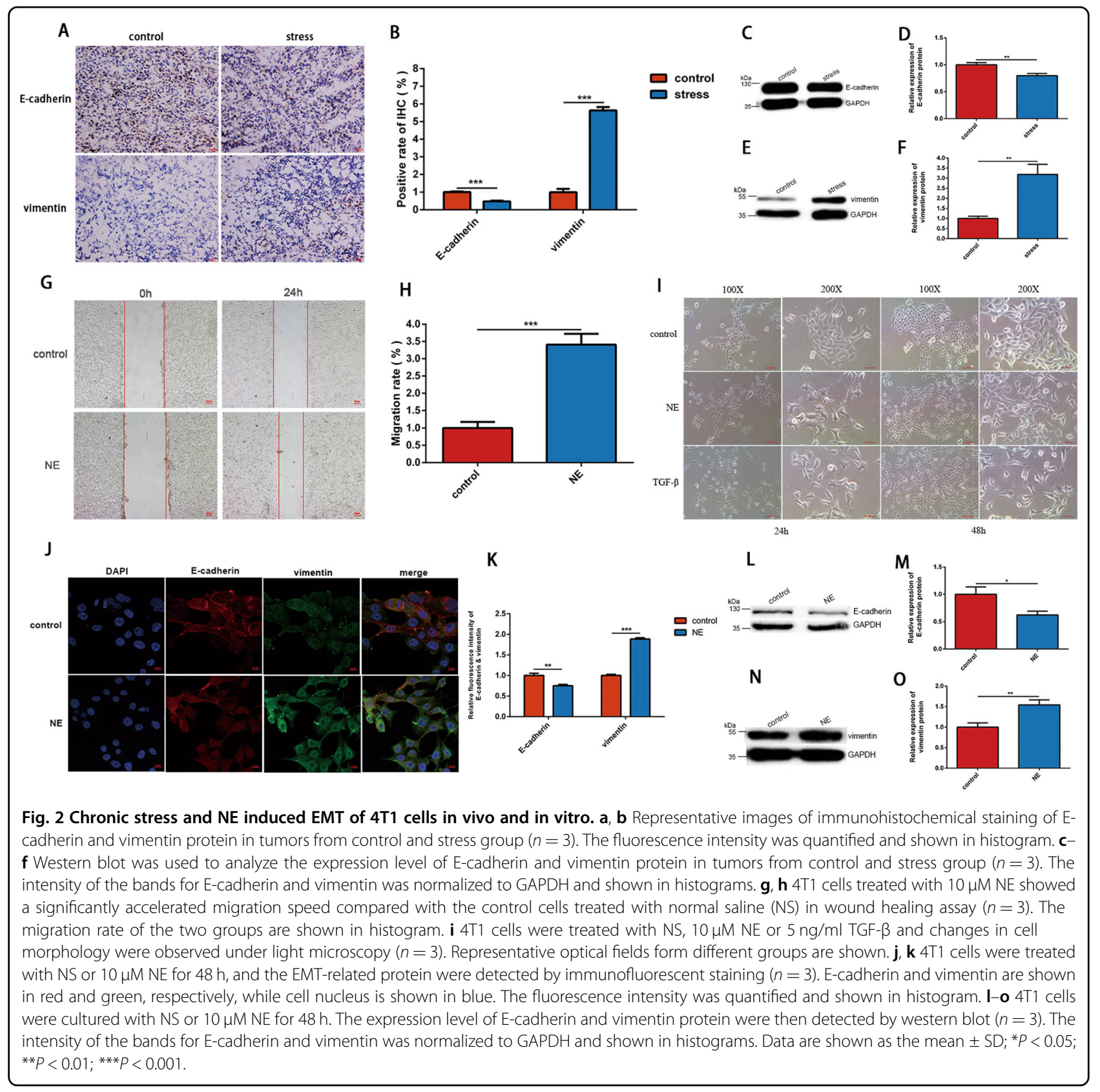

injected intratumorally in 4T1 tumor bearing mice for silencing the miR-337-3p. We observed that the tumor growth curves of each group were similar (Fig. 5a). Furthermore, there was no significant difference in the size and weight (Fig. 5b, c) of the tumors removed from each group. However, we found that the number of lung metastases in miR-337-3p-anta group (injected with miR337-3p antagomir intratumorally) was significantly increased compared to that in NS group (injected with normal saline intratumorally) and $\mathrm{NC}$-anta group (injected with negative control for antagomir intratumorally) (Fig. 5d, e). Furthermore, agomir was injected intratumorally to overexpress miR-337-3p in 4T1 tumor bearing mice under chronic restraint stress. We found that there was no significant difference in the tumor growth curve, tumor volume and tumor weight in each group (Fig. $5 \mathrm{a}-\mathrm{C}$ ), while the number of lung metastases in stress + miR-337-3p-ago group (being restrained and injected with miR-337-3p agomir intratumorally) decreased compared to that in stress + NS group (being restrained and injected with normal saline intratumorally) and stress $+\mathrm{NC}$-ago group (being restrained and injected with negative control for agomir intratumorally) (Fig. 5d, e). These results indicated that miR-337-3p could inhibit 


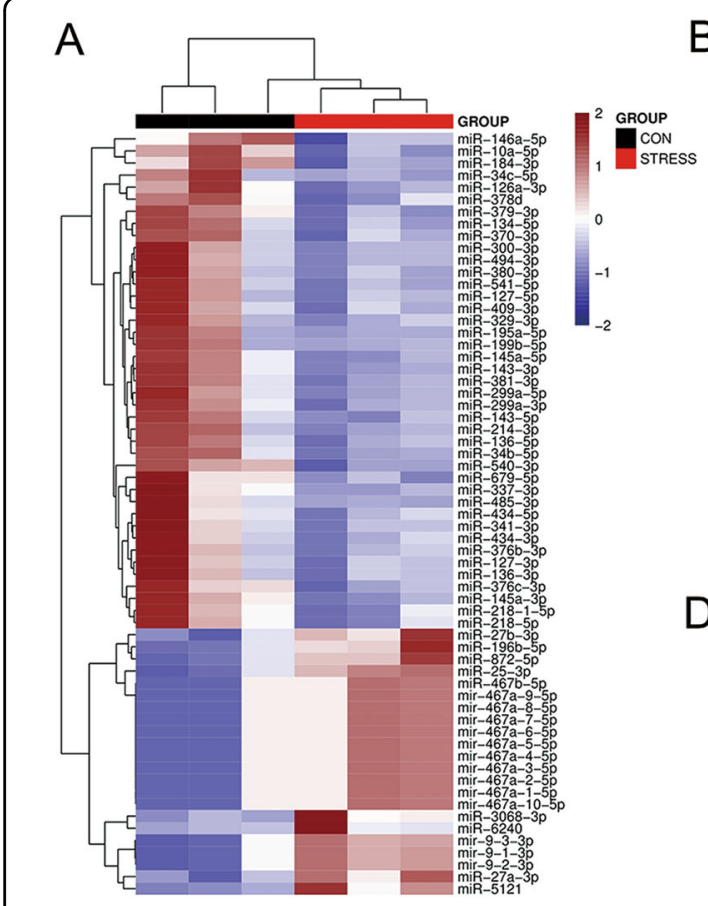

B

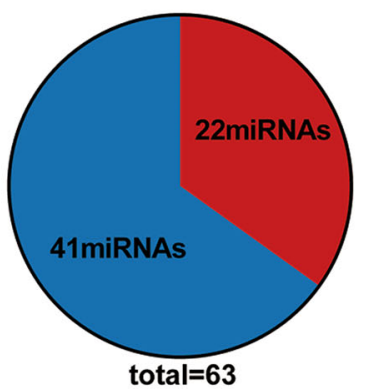

Upregulated

Downregulated

$\mathrm{D}$

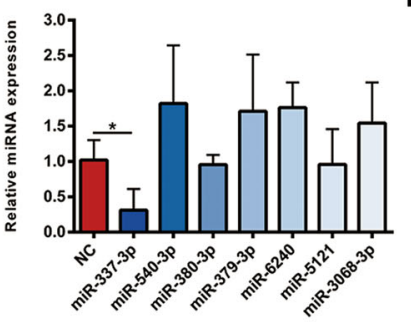

C

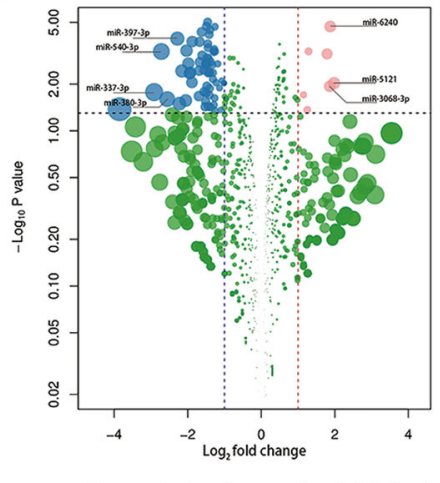

E

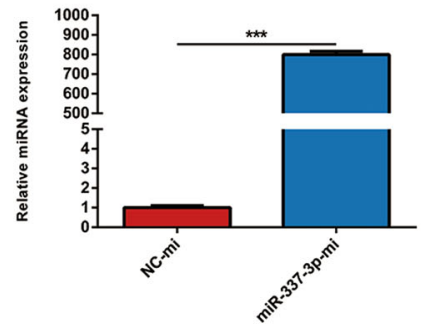

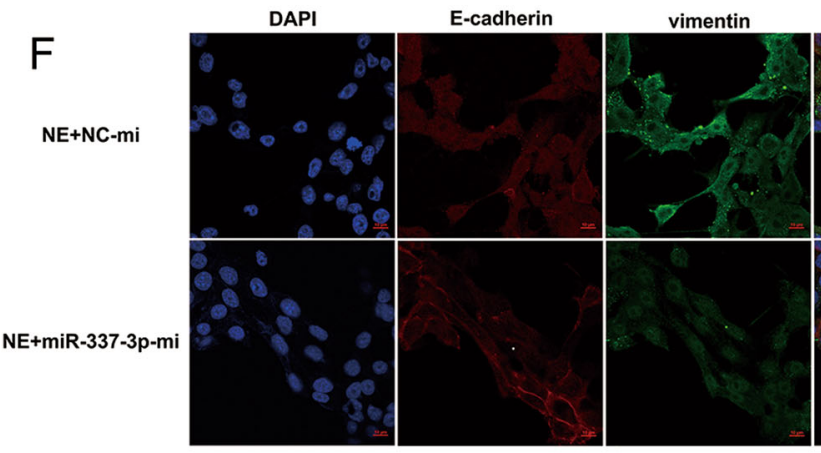

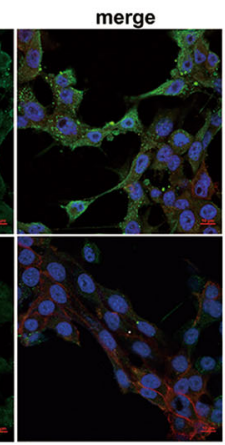

G
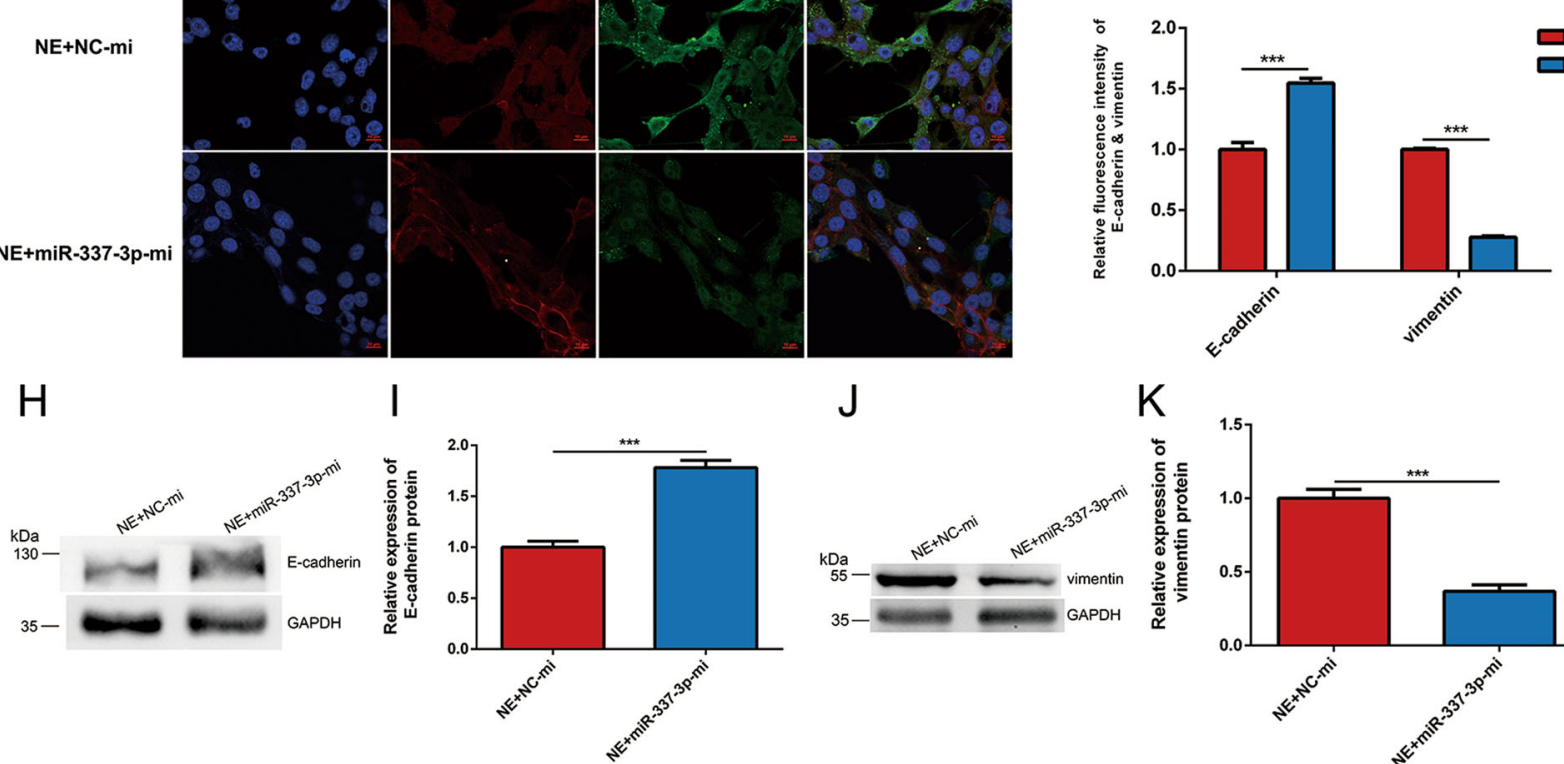

$J$

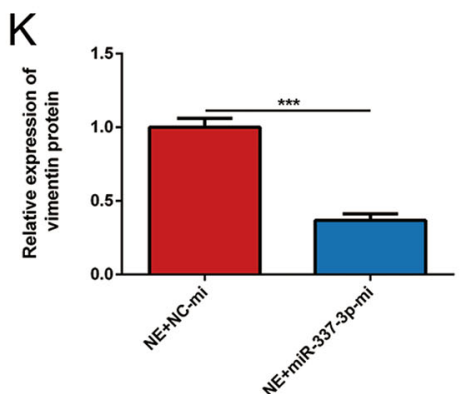

Fig. 3 miR-337-3p prevented EMT of 4 T1 cells induced by NE. a Heat map showing significantly changed $(P<0.05$, two-tailed Student's $t$-test) miRNAs between the control group and stress group. $\mathbf{b}$ Pie chart showing the number of upregulated and downregulated genes in Fig. 3a. $\mathbf{c}$ Volcano plots showing the specific seven miRNAs with $\| \mathrm{g} \mathrm{FCl}>1$ and $P<0.05$. d RT-qPCR analysis of microRNAs as listed in the figure were carried out in $4 \mathrm{~T} 1$ cells treated with $10 \mu \mathrm{M} \mathrm{NE}$ for $48 \mathrm{~h}(n=3)$. Relative miRNA levels were determined by the $\triangle \triangle$ Ct method using U6 for internal crossnormalization. e The transfection efficacy of the overexpression by miR-337-3p mimics in 4T1 cells was confirmed by RT-qPCR analysis ( $n=3$ ). Relative miRNA levels were determined by the $\triangle \Delta C$ t method using $U 6$ for internal cross-normalization. $\mathbf{f}, \mathbf{g}$ miR-337-3p-mi or NC-mi were transfected into 4T1 cells cultured with $10 \mu \mathrm{M} \mathrm{NE}$. After $48 \mathrm{~h}$, the EMT related protein were detected by immunofluorescent staining $(n=3)$. E-cadherin and vimentin are shown in red and green, respectively, while cell nucleus is shown in blue. The fluorescence intensity was quantified and shown in histogram. h-k miR-337-3p-mi or NC-mi were transfected into 4T1 cells cultured with $10 \mu \mathrm{M} \mathrm{NE}$. After $48 \mathrm{~h}$, the expression level of E-cadherin and vimentin were detected by western blot $(n=3)$. The intensity of the bands for E-cadherin and vimentin was normalized to GAPDH and shown in histograms. Data are shown as the mean $\pm \mathrm{SD} ;{ }^{*} P<0.05 ;{ }^{* *} P<0.001$. 


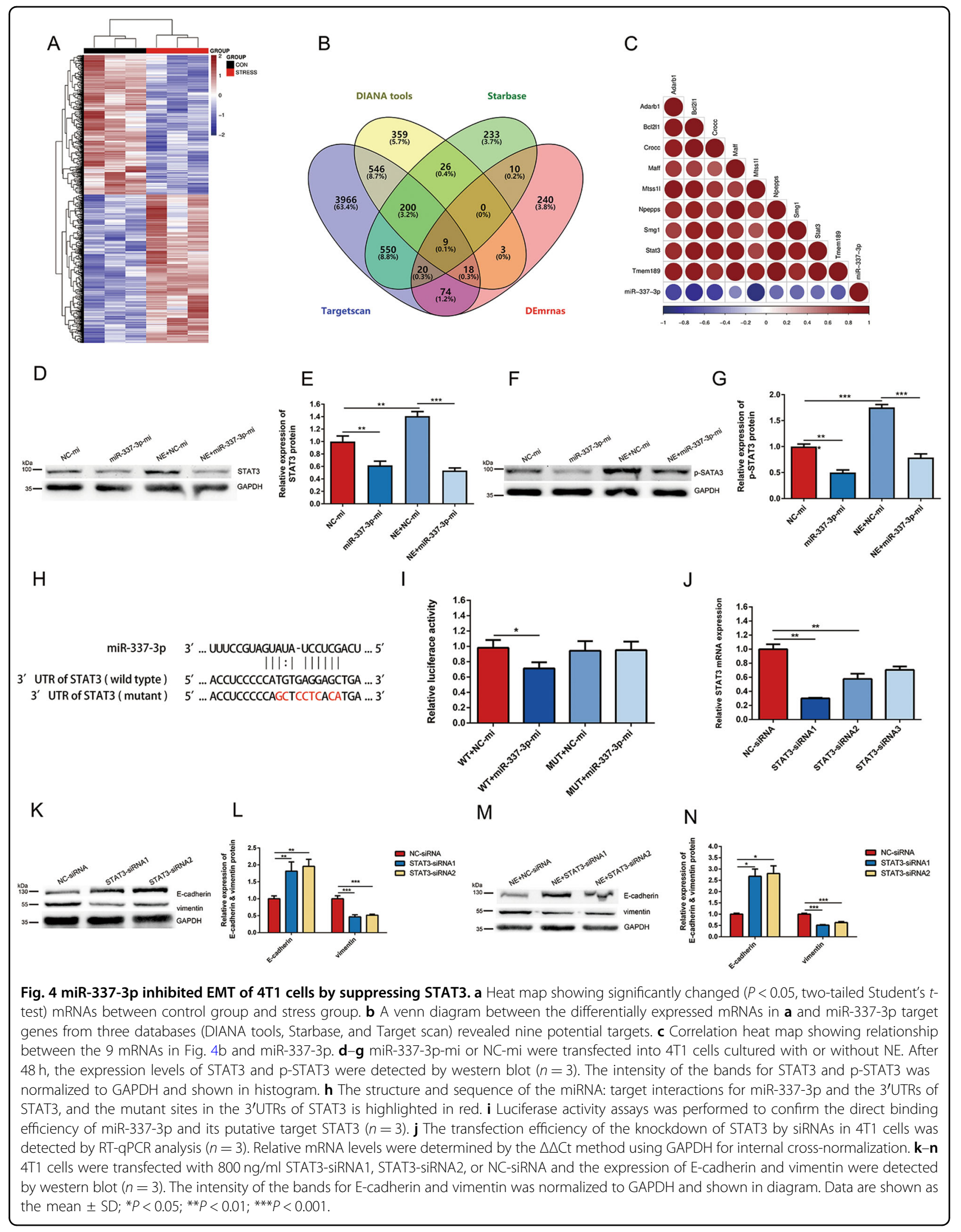




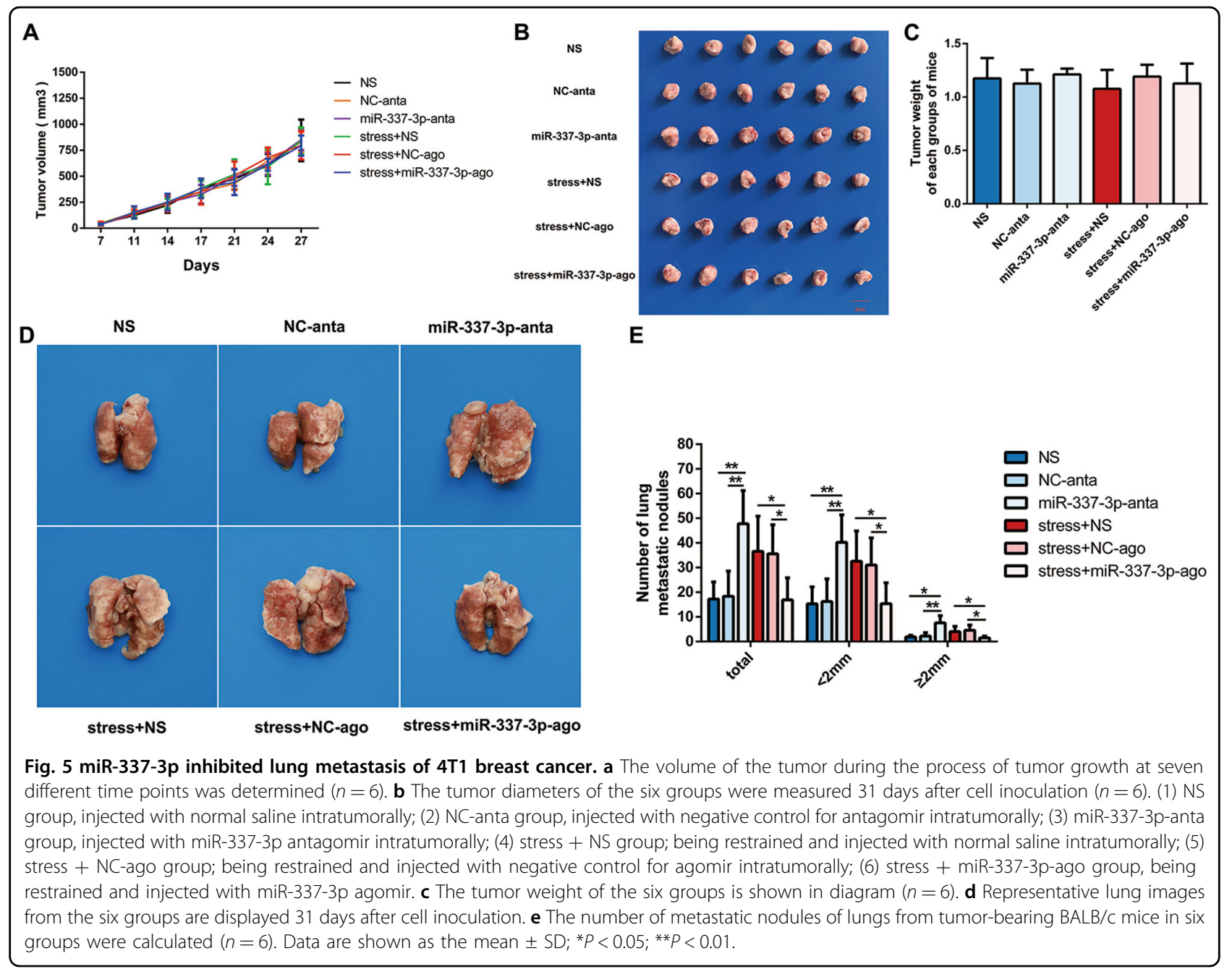

chronic stress associated lung metastasis without influencing the growth of $4 \mathrm{~T} 1$ xenograft.

To verify whether miR-337-3p inhibited EMT of 4T1 cancer cells in vivo, immunohistochemical staining and western blot analysis were used to detect the expression of related proteins. The results of immunohistochemical staining showed that silencing miR-337-3p by antagomir downregulated expression of E-cadherin while upregulated expression of vimentin. In addition, overexpression of miR-337-3p by agomir suppressed downregulation of E-cadherin and upregulation of vimentin induced by chronic restraint stress (Fig. 6a-d). Same results were obtained in western blot analysis (Fig. 6e-h).

To determine whether miR-337-3p regulated STAT3 and p-STAT3, we resorted to immunohistochemical staining and western blot analysis to detect the expression of the two proteins. The results from immunohistochemical staining indicated that silence of miR-337-3p by antagomir increased STAT3 and p-STAT3 expression. Moreover, the effect of stress on increasing STAT3 and
p-STAT3 expression was suppressed by overexpression of miR-337-3p (Fig. 6i-l). Same results were acquired in western blot analysis (Fig. $6 \mathrm{~m}-\mathrm{p}$ ). To sum up, the aforementioned results implied the critical role of miR-337-3p in regulating EMT and metastasis in vitro and in vivo, the proposed mechanism of which was shown in the schematic representation (Fig. 7).

\section{Discussion}

Patients with cancer tend to suffer chronic stress triggered by psychological disorders during the course of therapy and follow-up ${ }^{32}$. It has been well-reported that chronic stress could promote the release of stress-related hormone like NE through the activation of sympathetic nervous system or hypothalamic-pituitary-adrenal axis ${ }^{33}$. Prolonged exposure to stress-related hormones contributes to tumor growth, invasive capacity, and angiogenesis in various cancers ${ }^{10,34-36}$. Clinical and epidemiological studies also indicated that stress-related factors are related with cancer progression and poor 


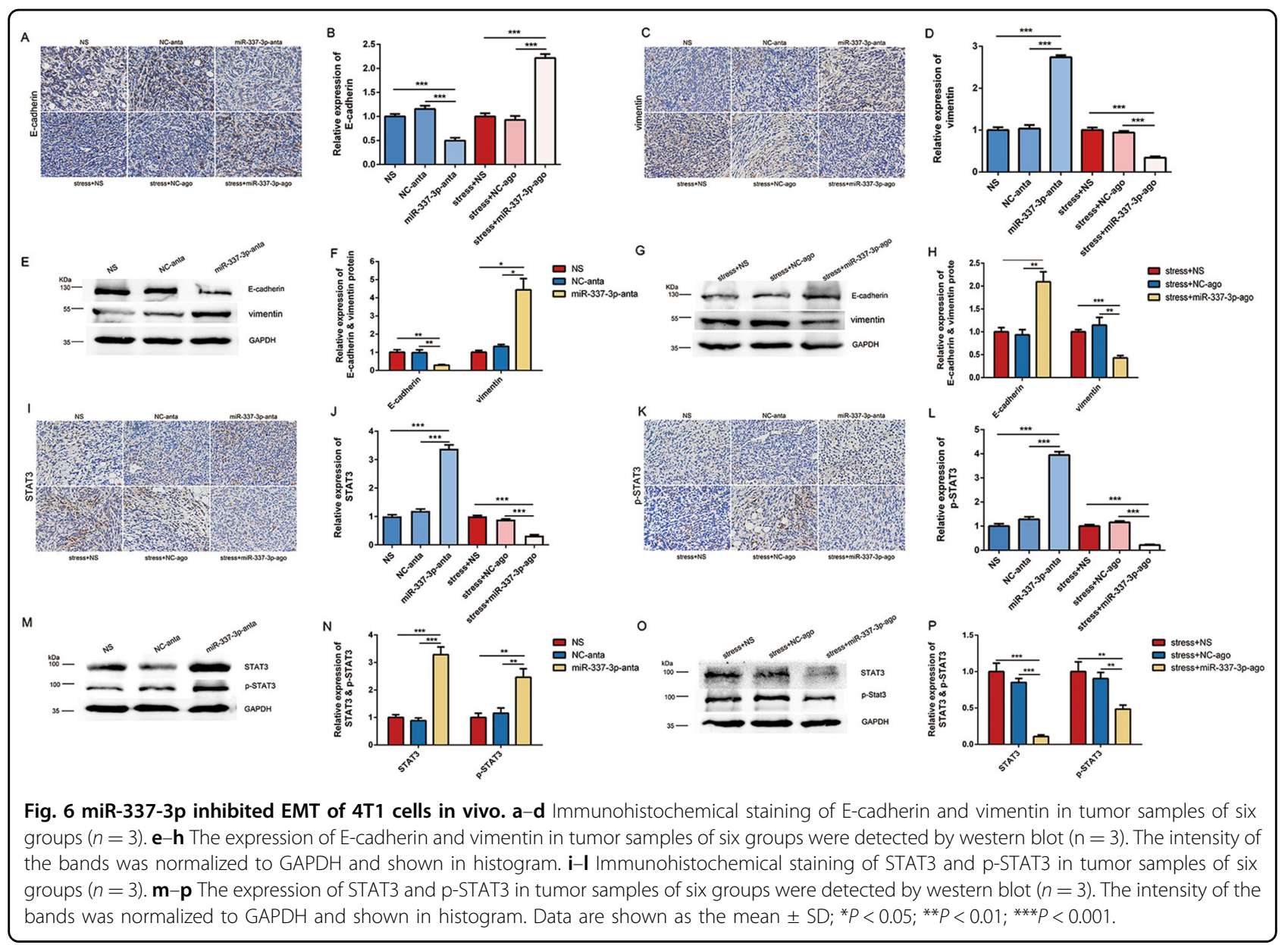

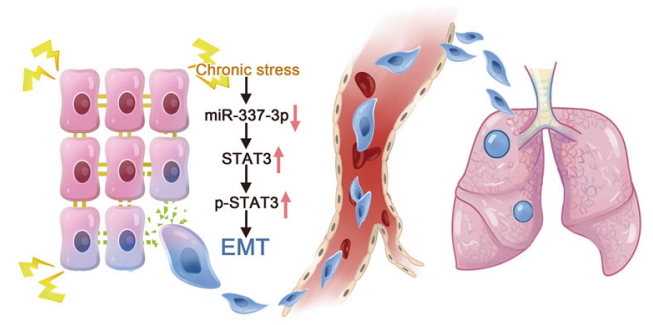

Fig. 7 Schematic representation of the proposed mechanism of chronic stress induced EMT and metastasis. Downregulated miR337-3p induced by chronic stress could futher activate STAT3 and promote EMT progression in breast cancer cells, which might contribute to lung metastasis.

outcomes ${ }^{37}$. A retrospective research indicated that psychological disorders among prostate cancer patients might be related to increasing invasive capability of tumor and metastasis ${ }^{38}$. In this study, we also found that chronic restraint stress contributed to lung metastasis of $4 \mathrm{~T} 1$ xenograft rather than tumor growth. Researchers exploring the effect of chronic stress on various cancers acquire contradictory results in the primary tumor growth, which may attribute to different cell lines and experimental design. However, they obtained similar results in chronic stress-related metastasis ${ }^{39-43}$.

Accumulating researches revealed the important role of EMT in the invasive capability and mobility of cancer cells $^{44}$. Activation of the EMT triggers a cascade of biological events to induce morphological changes in epithelial cells and bestows them metastatic phenotype, such as loss of cell junction and gain of mobility ${ }^{45}$. E-cadherin is a crucial protein participating in the cellular adhesions of epithelial cells and suppresses the metastasis of epithelial carcinomas ${ }^{46}$. Vimentin acts as a key factor in the final stage of EMT program and indicates the highly proliferative and invasive state of mesenchymal-like carcinoma cells ${ }^{47}$. Consistent with previous studies ${ }^{48,49}$, our study demonstrated that chronic stress and its related hormone NE downregulated the E-cadherin expression and upregulated the vimentin expression in vivo and in vitro. Additionally, as shown in Fig. 2, 4T1 cells cultured with NE became more elongated and separated compared to the control group and showed increased migration capacity. In all, the changes in molecular 
phenotype, cellular morphology and migration capabilities supported the development of EMT.

Previous studies validated that various cytokines, signaling pathway, and the interaction between tumor cells and extracellular matrix components all involved in the regulation of $\mathrm{EMT}^{50,51}$. Our previous work indicated two underlying signaling transduction pathways, namely $\beta$-AR/TGF- $\beta 1 / p-S m a d 3 /$ Snail and $\beta$-AR/TGF- $\beta 1 /$ HIF$1 \alpha /$ Snail, in the NE regulated EMT program in HT-29 and A549 cell lines ${ }^{52}$. In addition, NE could induce expression of human telomerase reverse transcriptase (hTERT) to promote ovarian cancer cell EMT and invasion by regulating ADBR2/PAK/Src/HIF- $1 \alpha$ and $\mathrm{c}-\mathrm{Myc}$ signaling pathway $^{53}$. In recent years, miRNAs are proved to be closely connected with the progression of $\mathrm{EMT}^{54}$. However, no research has confirmed the contributions of miRNA to the EMT induced by NE so far. In our study, next-generation sequencing and bioinformatic analysis were used to screen out differentially expressed miRNAs induced by chronic stress. Seven miRNAs were identified based on the criteria of $P<0.05$ and $\mid \log ^{2}$ (fold change) | $(|\lg \mathrm{FC}|)>1$. Among which, we selected miR-337-3p for further investigation, as its expression in 4T1 cells cultured with NE detected by RT-qPCR analysis was in consistent with the trend in bioinformatic prediction. The function of miR-337-3p in tumor progression has been illustrated by a few researchers. miR-337-3p acts as a suppressive role to modulate cell proliferation, EMT, and metastasis by targeting Capn 4 in clear cell renal cell carcinoma ${ }^{19}$. Additionally, miR-337-3p suppresses proliferation, migration, and invasion of cervical cancer cells by targeting Rap1A and the expression levels of miR-337-3p is related to better clinicopathological characteristics of cervical cancer ${ }^{55}$. We also found that overexpression of miR-337-3p by mimics reversed the NE induced EMT in 4T1 cells. Besides, miR-337-3p acted as a suppressor of EMT program in vivo. Taken together, miR-337-3p was an underlying regulator of chronic stress-related metastasis.

As a transcription factor in cytoplasmic, STAT3 could be activated in the cell signaling cascade in various cancers and acts as an important component in both EMT process and metastasis ${ }^{56-58}$. In our study, the results of bioinformatics analysis suggested that STAT3 might be a possible target gene of miR-337-3p. Furthermore, expression levels of STAT3 and p-STAT3 were upregulated by NE, and the phenomenon was reversed after overexpressing miR-337-3p in 4T1 cells. In vivo, STAT3 and p-STAT3 were also negatively regulated by miR-337$3 p$ in $4 \mathrm{~T} 1$ tumor models. We revealed the targeting relationship between miR-337-3p and STAT3 via dualluciferase reporter gene assay. miR-337-3p could negatively regulate the JAK2/STAT3 signaling cascade to promote cell proliferation and migration of hepatocellular carcinoma ${ }^{59}$. The sensitivity of nonsmall cell lung cancers to taxane also might be attributable to STAT3 targeted by miR-337-3p $p^{60}$. The direct targeting relationship between miR-337-3p and STAT3 contributes to the biological process of metastasis. Besides, we found that knockdown of STAT3 by siRNAs led to a reverse of EMT process, and suppressed NE induced EMT. The above results further supported the hypothesis that psychological disorders could promote metastasis.

To sum up, we demonstrated for the first time the involvement of miR-337-3p and its target gene STAT3 in regulating EMT program of breast cancer under chronic stress in vivo and in vitro. Further investigation of the mechanism underlying the metastasis effects of psychological disorders in cancer patients is warranted. The influence of psychological factors on tumor progression of patients requires more attention in clinical.

\section{Materials and methods Cell culture}

All cell lines used for experiments were acquired from American Type Culture Collection (Manassas, VA, USA). RPMI-1640 medium (Gibco, Carlsbad, CA, USA) was used for cultivating the 4T1 tumor cells and Dulbecco's modified Eagle's medium (Gibco, Carlsbad, CA, USA) was used for incubating the HEK-293T cells, both mediums containing 10\% FBS. All cells were placed in an incubator with $5 \% \mathrm{CO}_{2}$ and $95 \%$ saturated humidity at $37^{\circ} \mathrm{C}$, and the medium was changed every two days.

\section{Mice model}

The 6-weeks-old BALB/c female mice were provided by Beijing Huafukang Bioscience (Beijing, China). To induce tumors, $1 \times 10^{5} 4 \mathrm{~T} 1$ cells suspended in $100 \mu \mathrm{l}$ of saline injected subcutaneously into the right breast region of each mouse.

Mice model 1: Mice model 1 were used for exploring the effect of chronic restraint stress on tumor. In this model, ten mice were randomly divided into control group and stress group 7 days after 4T1 cell inoculation. Mice in stress group were restrained in drafty $50 \mathrm{ml}$ conical bottom centrifuge tubes $2 \mathrm{~h}$ daily for consecutive 28 days, while mice in control group were free to move. The theory foundation of chronic restraint stress model were detailedly elucidated in our previous work ${ }^{61}$.

Mice model 2: Mice model 2 were used for verifying the effect of miR-337-3p on metastasis and EMT in vivo. In this model, 36 mice were randomly divided into six groups according to different treatments 7 days after 4T1 cell inoculation: (1) NS group, injected with normal saline intratumorally; (2) NC-anta group, injected with negative control for antagomir (Ribobio, Guangzhou, China) intratumorally; (3) miR-337-3p-anta group, injected with miR-337-3p antagomir (Ribobio, Guangzhou, China) 
intratumorally; (4) stress + NS group; being restrained and injected with normal saline intratumorally; (5) stress $+\mathrm{NC}$-ago group; being restrained and injected with negative control for agomir (Ribobio, Guangzhou, China) intratumorally; (6) stress + miR-337-3p-ago group, being restrained and injected with miR-337-3p agomir (Ribobio, Guangzhou, China) intratumorally. The mice were restrained $2 \mathrm{~h}$ daily and the intratumor injection was administrated twice a week for 7 times in total. Tumors were measured using vernier caliper twice a week, and volume was calculated using the method described by Seiji Naito ${ }^{62}$. In order to count metastases, lungs were soaked in saline solution and cleaned. Photographs of the tumors and lungs were taken and the number of nodules on the surface of lungs was quantified. All animal experiments were carried out in conformity with the Guide of the Animal Care and Use Committee of West China Hospital, Sichuan University, China.

\section{Specimens and sequencing}

For the next-generation sequencing on Illumina Hiseq 2500/2000 platform, miRNAs and mRNAs were extracted from tumor tissues of control group and stress group in mice model 1. After removing adapter sequences, collapsing reads with the same sequence, and reading with less than 15 bases in length from raw data, clean data were obtained and analyzed based on the miRDeep2(2.0.0.8). miRNA and mRNA reads were mapped to mice miRNA precursors from miRBASE release 22.1 using miRDeep2 allowing zero mismatches. Mapped reads were then quantified by miRDeep2. Analysis of the differentially expressed miRNAs and mRNAs between the control group and stress group were performed by the DEseq2 $\mathrm{R}$ package. The significance threshold was set as $P<0.05 \& \mid$ $\log ^{2}$ (fold change) $\mid>1$ in this research.

\section{miRNA, siRNA and plasmid transfections}

$5 \times 10^{4}$ cells/well $4 \mathrm{~T} 1$ cells were plated onto 12 -well plate for $24 \mathrm{~h}$ in a humidified incubator of $5 \% \mathrm{CO}_{2}$ and $37^{\circ} \mathrm{C}$. The transfection of all miRNA mimics and siRNAs were performed using Lipofectamine ${ }^{\mathrm{TM}}$ RNAiMAX Transfection Reagent (Invitrogen, Carlsbad, California, USA) in guidance with the standard protocol, and reporter gene plasmid was transfected through the use of Lipofectamine 3000 reagent (Invitrogen, Carlsbad, California, USA). The cells were collected for next experiments after $6 \mathrm{~h}$ of transfection.

\section{Wound healing assay}

$5 \times 10^{5}$ cells/well $4 \mathrm{~T} 1$ cells were planted into 6-well plates for incubation at $37^{\circ} \mathrm{C}$. We used a sterilized tip of transfer liquid gun to gently draw lines on the plate when cells covered the bottom of the plate, and the width of all scratches were required to be about the same. After removing cell culture fluid and washing the plate with PBS buffer for three times to wash away cell debris produced by scratches, cells were photographed $(0 \mathrm{~h})$. Next, serum-free medium was added into the plate for blocking cell proliferation and the cells were photographed at the same points again after incubation at $37^{\circ} \mathrm{C}$ for $24 \mathrm{~h}$. The OLYMPUS inverted microscope (Tokyo, Japan) was used to take photos the scratches and the area of cells migrating to the scratched area was estimated by using Image J software.

\section{Immunohistochemistry}

After being incubated at $65^{\circ} \mathrm{C}$ for $30 \mathrm{~min}$, the sections were subjected to deparaffination by using xylene, transferred to gradient ethyl alcohol (100\% ethanol, $5 \mathrm{~min}$; $95 \%$ ethanol, $2 \mathrm{~min}$; 90\% ethanol, $2 \mathrm{~min}$; $80 \%$ ethanol, $2 \mathrm{~min}$ ), washed with PBS for $2 \mathrm{~min}$. Then, the sections were boiled with $0.01 \mathrm{M}$ citric acid buffer ( $\mathrm{pH}$ 6.0) in a heated bath of $97^{\circ} \mathrm{C}$ for $40 \mathrm{~min}$ to repair antigen, soaked in 3\% $\mathrm{H}_{2} \mathrm{O}_{2}$ for 30 min for quenching the exogenous peroxidase. After blocking with goat serum for $30 \mathrm{~min}$, the sections were exposed with the following primary antibodies, rabbit antimouse antibody to E-cadherin (Proteintech, Wuhan, China) and chicken antimouse antibody to vimentin (Abcam, Cambridge, UK) at $37^{\circ} \mathrm{C}$ for $2 \mathrm{~h}$ and washed with PBS. Subsequent incubation was performed with the secondary antibodies labeled with horseradish peroxidase, goat antichicken antibody to immunoglobulin Y (IgY) (Abcam, Cambridge, UK) and goat antirabbit antibody to immunoglobulin G (IgG) (Jackson, Philadelphia, Pennsylvania, USA), at $37^{\circ} \mathrm{C}$ for $30 \mathrm{~min}$. The sections were then counterstained with hematoxylin (Bioss, Beijing, China). After sealing with Clear-Mount (Head biotechnology, Beijing, China), the slides were observed under an electron microscope (Zeiss, Oberkochen, Germany) with images subsequently obtained.

\section{Immunofluorescence}

$2 \times 10^{4}$ cells/well $4 \mathrm{~T} 1$ cells were inoculated in a 24 -well culture plate with cell slides placed at the bottom in advance, so that the cells expand to $60-70 \%$ before experiment. The cells on the slides were washed by PBS for three times and then fixed with $500 \mu \mathrm{l} 4 \%$ polyformaldehyde at room temperature for $15 \mathrm{~min}$. After that, pre-cooled $0.2 \%$ Triton X-100 diluted in PBS was used to permeabilize the cells for $5 \mathrm{~min}$. Then, the cells in each well were blocked with $500 \mu$ l goat serum containing $0.3 \%$ Triton X-100 at room temperature for an hour. After removal of the goat serum, the cells were covered with the mixed primary antibodies of E-cadherin (CST, Boston, Massachusetts, USA) and vimentin (Abcam, Cambridge, UK) overnight at $4{ }^{\circ} \mathrm{C}$ under dark conditions, and the two primary antibodies were coupled with Alexa Fluor ${ }^{\circledR} 594$ and Alexa Fluor ${ }^{\circledR} 488$, respectively. Afterwards, cells were 
washed three times using PBS, stained with DAPI staining solution (Invitrogen, Camarillo, CA, USA) for $10 \mathrm{~min}$, washed, and sealed with BrightMount (Abcam, Cambridge, UK). Cells on the slides were observed by using a fluorescence confocal microscope (Zeiss, Oberkochen, Germany) with images subsequently obtained.

\section{Real-time quantitative polymerase chain reaction (RT-qPCR) analysis}

miRNA was extracted with a miRcute miRNA extraction and separation kit (TIANGEN, Beijing, China) while total RNA was extracted with Trizol (Takara, Shiga, Japan). After measuring the concentration, miRNA and mRNA was reversely transcribed into cDNA with a miRcute Plus miRNA First-Strand cDNA Kit (TIANGEN, Beijing, China) and PrimeScript RT reagent Kit with gDNA Eraser (Takara, Shiga, Japan), respectively. Expression analysis of miRNA and mRNA was performed on a LightCycler 96 System (Roche, Basel, Switzerland) by using miRcute Plus miRNA qPCR Kit (TIANGEN, Beijing, China) and TB Green ${ }^{\circledR}$ Premix Ex Taq ${ }^{\mathrm{TM}}$ II (Takara, Shiga, Japan), respectively. All primers for miRNA were ordered form GeneCopoeia Inc. (Guangzhou, China) and primers for mRNA were synthesized by GenePharma Co. (Shanghai, China), the sequences of all primers are shown in Table S1. U6 and GAPDH were used as the internal control for miRNAs and mRNA, respectively. The $\mathrm{Ct}$ value was recorded and U6 was served as the internal control for miRNA, while glyceraldehyde-3-phosphate dehydrogenase (GAPDH) serving as the internal control for STAT3. The $\Delta \Delta$ Ct method was used for calculating the relative expression of genes.

\section{Western blots}

The total protein from tumor tissues or cells was extracted with RIPA lysis buffer (Beyotime, Shanghai, China) containing inhibitors of protease and phosphatase. The concentration of each protein sample was detected by using BCA protein assay kit (Solarbio, Beijing, China) in line with the manufacturer's recommendation. For electrophoresis, same quantity of protein lysate was added to each lane and then transferred onto the Polyvinylidene fluoride (PVDF) membrane (Bio-Rad, California, USA). Next, the membrane was soaked into the $5 \%$ defatted milk for $1 \mathrm{~h}$ in order to block the non-specific sites. Then, the membrane was covered overnight at $4{ }^{\circ} \mathrm{C}$ with the following primary antibodies: rabbit antimouse antibodies against E-cadherin (Proteintech, Wuhan, China), vimentin (Bioss, Beijing, China), STAT3 (Abcam, Cambridge, UK), p-STAT3 (Abcam, Cambridge, UK) and mouse antimouse antibody GAPDH (ZenBio, Chengdu, China). After washing five times by Tris-Buffered Saline with Tween-20 (TBST), the membranes were immersed into TBST with the secondary antibody, HRP-labeled goat antirabbit IgG
(ZenBio, Chengdu, China) and goat antimouse IgG (ZenBio, Chengdu, China), respectively, for $1 \mathrm{~h}$. After washing with TBST for three times, the membrane was treated with the Immobilon western HRP Substrate (Millipore, Bedford, MA, USA) and the bands were detected by iBright CL1000 Imaging System (Invitrogen, Carlsbad, California, USA). The relative intensity of each blot was assessed and analyzed by using the Image J Software.

\section{Dual-luciferase reporter gene assay}

The pEZX-FR02-WT-STAT3 (STAT3-WT) containing the $3^{\prime}$-UTR region and pEZX-FR02-Mut-STAT3 (STAT3-Mut) containing the mutated binding site of miR-337-3p were constructed (GeneCopoeia, Guangzhou, China). In guidance with protocol of previous publication $^{63,64}$, NC mimic and miR-337-3p mimic were cotransfected with STAT3-WT or STAT3-Mut into HEK$293 \mathrm{~T}$ cells. After $48 \mathrm{~h}$ of incubation, cells were subjected to measure the luciferase activity on the basis of specification of the dual-luciferase reporter gene assay kit (Beyotime, Shanghai, China). The fluorescence intensity was detected using SpectraMAX i3x Ptatform (Molecular Devices, California, USA).

\section{Statistical analysis}

The statistical analyses of all experimental data were carried out using GraphPad Prism software version 6.0. (GraphPad Software, Inc., California, USA). All measurement data were presented in a form of mean \pm SD. Student's $t$-test (two-sided) analyses were performed between each two groups. $P<0.05$ was regarded as an indication of significant differences.

\section{Acknowledgements \\ This work was supported by National Natural Science Foundation of China (Grant Numbers: 81572853 and 81902685 ). The authors gratefully thank Dr. Deng at Jacobi Medical center for his helpful comments on language proficiency that substantially improved the manuscript.}

Conflict of interest

The authors declare that they have no conflict of interest.

\section{Publisher's note}

Springer Nature remains neutral with regard to jurisdictional claims in published maps and institutional affiliations.

Supplementary Information accompanies this paper at (https://doi.org/ 10.1038/s41419-020-02981-1).

Received: 1 January 2020 Revised: 29 August 2020 Accepted: 3 September 2020

Published online: 15 September 2020

\footnotetext{
References

1. Andrykowski, M. A., Lykins, E. \& Floyd, A. Psychological health in cancer survivors. Semin Oncol. Nurs. 24, 193-201 (2008).
} 
2. Powell, N. D., Tarr, A. J. \& Sheridan, J. F. Psychosocial stress and inflammation in cancer. Brain Behav. Immun. 30, S41-S47 (2013).

3. Antoni, M. H. Psychosocial intervention effects on adaptation, disease course and biobehavioral processes in cancer. Brain Behav. Immun. 30, S88-S98 (2013).

4. Medeiros, B. \& Allan, A. L. Molecular mechanisms of breast cancer metastasis to the lung: clinical and experimental perspectives. Int. J. Mol. Sci. https://doi. org/10.3390/ijms20092272 (2019).

5. Bray, F. et al. Global cancer statistics 2018: Globocan estimates of incidence and mortality worldwide for 36 cancers in 185 countries. Cancer J. Clin. 68, 394-424 (2018).

6. Ji, H. et al. Oxytocin involves in chronic stress-evoked melanoma metastasis via $\beta$-arrestin 2-mediated erk signaling pathway. Carcinogenesis 40, 1395-1404 (2019).

7. Chen, H. et al. Chronic psychological stress promotes lung metastatic colonization of circulating breast cancer cells by decorating a pre-metastatic niche through activating $\beta$-adrenergic signaling. J. Pathol. 244, 49-60 (2018).

8. Hermes, G. L. et al. Social isolation dysregulates endocrine and behavioral stress while increasing malignant burden of spontaneous mammary tumors. Proc. Natl Acad. Sci. USA 106, 22393-22398 (2009).

9. Madden, K. S., Szpunar, M. J. \& Brown, E. B. Early impact of social isolation and breast tumor progression in mice. Brain Behav. Immun. 30, S135-S141 (2013).

10. Chang, A. et al. Beta2-adrenoceptors on tumor cells play a critical role in stressenhanced metastasis in a mouse model of breast cancer. Brain Behav. Immun. 57, 106-115 (2016).

11. Campbell, J. P. et al. Stimulation of host bone marrow stromal cells by sympathetic nerves promotes breast cancer bone metastasis in mice. PLoS Biol. 10, e1001363 (2012).

12. Berhili, S. et al. Associated factors with psychological distress in moroccan breast cancer patients: a cross-sectional study. Breast 31, 26-33 (2017).

13. Mittal, V. Epithelial mesenchymal transition in tumor metastasis. Annu. Rev. Pathol. 13, 395-412 (2018)

14. Kalluri, R. \& Weinberg, R. A. The basics of epithelial-mesenchymal transition. J. Clin. Invest. 119, 1420-1428 (2009).

15. Pang, $H$. et al. Prognostic values of osteopontin-c, e-cadherin and $\beta$-catenin in breast cancer. Cancer Epidemiol. 37, 985-992 (2013).

16. Miska, E. A. How micrornas control cell division, differentiation and death. Curr. Opin. Genet. Dev. 15, 563-568 (2005).

17. Fabian, M. R., Sonenberg, N. \& Filipowicz, W. Regulation of mrna translation and stability by micrornas. Annu. Rev. Biochem. 79, 351-379 (2010).

18. Khaled, N. \& Bidet, Y. New insights into the implication of epigenetic alterations in the emt of triple negative breast cancer. Cancers. https://doi.org/ 10.3390/cancers11040559 (2019).

19. Zhuang, Q. et al. Mir-337-3p suppresses the proliferation and metastasis of clear cell renal cell carcinoma cells via modulating capn4. Cancer Biomark. 23, 515-525 (2018).

20. Banerjee, $\mathrm{K}$. \& Resat, $\mathrm{H}$. Constitutive activation of stat3 in breast cancer cells: a review. Int. J. Cancer 138, 2570-2578 (2016).

21. Li, J., Wang, T. \& Jiang, X. F. Inhibition of mir-337-3p involved in the protection of $\operatorname{cocl}(2)$-induced injury in pc12 cells via activating jak2/stat3 signaling pathway. J. Cell Biochem. 120, 19076-19086 (2019).

22. Du, L. et al. Mir-337-3p and its targets stat3 and rap1a modulate taxane sensitivity in non-small cell lung cancers. PLoS ONE 7, e39167 (2012).

23. Olejniczak, M., Kotowska-Zimmer, A. \& Krzyzosiak, W. Stress-induced changes in mirna biogenesis and functioning. Cell Mol. Life Sci. 75, 177-191 (2018).

24. Shen, $C$. et al. Downregulation of mir-541 induced by heat stress contributes to malignant transformation of human bronchial epithelial cells via hsp27. Environ. Res. 184, 108954 (2020).

25. Ma, X., Wang, M., Yin, T., Zhao, Y. \& Wei, X. Myeloid-derived suppressor cells promote metastasis in breast cancer after the stress of operative removal of the primary cancer. Front. Oncol. 9, 855 (2019).

26. Berger, M. F. \& Mardis, E. R. The emerging clinical relevance of genomics in cancer medicine. Nat. Rev. Clin. Oncol. 15, 353-365 (2018).

27. Coenen-Stass, A. M. L. et al. Evaluation of methodologies for microrna biomarker detection by next generation sequencing. RNA Biol. 15, 1133-1145 (2018).

28. Cui, Y. et al. Differential expression of mirna in rat myocardial tissues under psychological and physical stress. Exp. Ther. Med. 7, 901-906 (2014).

29. Du, L. et al. Mir-337-3p and its targets stat3 and rap1a modulate taxane sensitivity in non-small cell lung cancers. PLoS ONE 7, e39167 (2012).
30. Li, Q., Huang, Q., Cheng, S., Wu, S. \& Hou, J. Circ_znf124 promotes non-small cell lung cancer progression by abolishing mir-337-3p mediated downregulation of jak2/stat3 signaling pathway. Cancer Cell Int. 19, 291 (2019).

31. Huang, Y. et al. Znas@sio2 nanoparticles as a potential anti-tumor drug for targeting stemness and epithelial-mesenchymal transition in hepatocellular carcinoma via shp-1/jak2/stat3 signaling. Theranostics 9, 4391-4408 (2019).

32. Cordova, M. J., Riba, M. B. \& Spiegel, D. Post-traumatic stress disorder and cancer. Lancet Psychiatry 4, 330-338 (2017).

33. Stratakis, C. A. \& Chrousos, G. P. Neuroendocrinology and pathophysiology of the stress system. Ann. N. Y. Acad. Sci. 771, 1-18 (1995).

34. Xie, $\mathrm{H}$. et al. Chronic stress promotes oral cancer growth and angiogenesis with increased circulating catecholamine and glucocorticoid levels in a mouse model. Oral. Oncol. 51, 991-997 (2015).

35. Thaker, P. H. et al. Chronic stress promotes tumor growth and angiogenesis in a mouse model of ovarian carcinoma. Nat. Med. 12, 939-944 (2006).

36. Kim-Fuchs, C. et al. Chronic stress accelerates pancreatic cancer growth and invasion: a critical role for beta-adrenergic signaling in the pancreatic microenvironment. Brain Behav. Immun. 40, 40-47 (2014).

37. Moreno-Smith, M., Lutgendorf, S. K. \& Sood, A. K. Impact of stress on cancer metastasis. Future Oncol. 6, 1863-1881 (2010).

38. Cheng, $Y$. Depression promotes prostate cancer invasion and metastasis via a sympathetic-camp-fak signaling pathway. Oncogene 37, 2953-2966 (2018).

39. $\mathrm{Wu}, \mathrm{W}$. et al. Social isolation stress impairs the resistance of mice to experimental liver metastasis of murine colon 26-15 carcinoma cells. Biol. Pharm. Bull. 24, 772-776 (2001).

40. Lin, Q. et al. Effect of chronic restraint stress on human colorectal carcinoma growth in mice. PLOS ONE 8, e61435 (2013).

41. Le, C. P. et al. Chronic stress in mice remodels lymph vasculature to promote tumour cell dissemination. Nat. Commun. 7, 10634 (2016).

42. Budiu, R. A. et al. Restraint and social isolation stressors differentially regulate adaptive immunity and tumor angiogenesis in a breast cancer mouse model. Cancer Clin. Oncol. 6, 12-24 (2017)

43. Arranz, A. et al. The impact of stress on tumor growth: peripheral crf mediates tumor-promoting effects of stress. Mol. Cancer 9, 261 (2010).

44. Lu, W. \& Kang, Y. Epithelial-mesenchymal plasticity in cancer progression and metastasis. Dev. Cell 49, 361-374 (2019).

45. Felipe Lima, J., Nofech-Mozes, S., Bayani, J. \& Bartlett, J. M. Emt in breast carcinoma-a review. J. Clin. Med. https://doi.org/10.3390/jcm5070065 (2016).

46. Matos, M. L. et al. Identification of a novel human e-cadherin splice variant and assessment of its effects upon emt-related events. J. Cell Physiol. 232 1368-1386 (2017).

47. Wu, S., Du, Y., Beckford, J. \& Alachkar, H. Upregulation of the emt marker vimentin is associated with poor clinical outcome in acute myeloid leukemia. J. Transl. Med. 16, 170 (2018).

48. Zhang, J. et al. Norepinephrine induced epithelial-mesenchymal transition in ht-29 and a549 cells in vitro. J. Cancer Res. Clin. Oncol. 142, 423-435 (2016).

49. Shan, T. et al. Novel regulatory program for norepinephrine-induced epithelialmesenchymal transition in gastric adenocarcinoma cell lines. Cancer Sci. 105, 847-856 (2014)

50. Lamouille, S., Xu, J. \& Derynck, R. Molecular mechanisms of epithelialmesenchymal transition. Nat. Rev. Mol. Cell Biol. 15, 178-196 (2014).

51. Gonzalez, D. M. \& Medici, D. Signaling mechanisms of the epithelialmesenchymal transition. Sci. Signal. 7, re8 (2014).

52. Zhang, Jie et al. Norepinephrine induced epithelial-mesenchymal transition in ht-29 and a549 cells in vitro. J. Cancer Res. Clin. Oncol. 142, 423-435 (2015).

53. Choi, M. J. et al. Htert mediates norepinephrine-induced slug expression and ovarian cancer aggressiveness. Oncogene 34, 3402-3412 (2015).

54. Abba, M. L., Patil, N., Leupold, J. H. \& Allgayer, H. Microrna regulation of epithelial to mesenchymal transition. J. Clin. Med. https://doi.org/10.3390/ jcm5010008 (2016).

55. Cao, X. M. Role of mir-337-3p and its target rap1a in modulating proliferation, invasion, migration and apoptosis of cervical cancer cells. Cancer Biomark. 24 257-267 (2019).

56. Zhang, $\mathrm{H}$. et al. Mir-7, inhibited indirectly by lincrna hotair, directly inhibits setdb1 and reverses the emt of breast cancer stem cells by downregulating the stat3 pathway. Stem Cells 32, 2858-2868 (2014).

57. Wang, Y. et al. Tgf- $\beta$-induced stat3 overexpression promotes human head and neck squamous cell carcinoma invasion and metastasis through malat1/mir30a interactions. Cancer Lett. 436, 52-62 (2018). 
58. Kim, M. J., Lim, J., Yang, Y., Lee, M. S. \& Lim, J. S. N-myc downstream-regulated gene 2 (ndrg2) suppresses the epithelial-mesenchymal transition (emt) in breast cancer cells via stat3/snail signaling. Cancer Lett. 354, 33-42 (2014).

59. Zuo, X. L. et al. Mir-337-3p suppresses the proliferation and invasion of hepatocellular carcinoma cells through targeting jak2. Am. J. Cancer Res. 8, 662-674 (2018)

60. Du, L. et al. Mir-337-3p and its targets stat3 and rapla modulate taxane sensitivity in non-small cell lung cancers. PLOS ONE 7, e39167 (2012).

61. Liu, J. et al. The effect of chronic stress on anti-angiogenesis of sunitinib in colorectal cancer models. Psychoneuroendocrinology 52, 130-142 (2015).
62. Naito, S., Eschenbach, A. C., Von, Giavazzi, R. \& Fidler, I. J. Growth and metastasis of tumor cells isolated from a human renal cell carcinoma implanted into different organs of nude mice. Cancer Res. 46, 4109 (1986).

63. Zhang, L., Gao, J. \& Cui, S. Mir-21 is involved in norepinephrine-mediated rat granulosa cell apoptosis by targeting smad7. J. Mol. Endocrinol. 58, 199-210 (2017).

64. Lu, Z. et al. Microrna-17 functions as an oncogene by downregulating smad3 expression in hepatocellular carcinoma. Cell Death Dis. 10, 723 (2019). 